УДК 621.396 .96

\title{
АДАПТИВНАЯ ФИЛЬТРАЦИЯ ПАРАМЕТРОВ ДВИЖЕНИЯ ИСТОЧНИКА РАДИОИЗЛУЧЕНИЯ НА ОСНОВЕ ТDОА-ИЗМЕРЕНИЙ СЕНСОРНОЙ СЕТИ ПРИ НАЛИЧИИ АНОМАЛЬНЫХ ИЗМЕРЕНИЙ
}

\author{
С. Я. ЖУК, И. О. ТОВКАЧ, Ю. Ю. РЕУТСКАЯ
}

Национальный технический университет Украиньл «Киевский политехнический институт им. Игоря Сикорского», Украина, Киев, 03056, пр-т Победы 37

\begin{abstract}
Аннотация. Методы на основе TDOA-измерений находят широкое применение для определения местоположения источника радиоизлучения с помощью беспроводных сенсорных сетей. В реальных условиях часто возникает необходимость учитывать наличие аномальных результатов измерений. Их появление означает существенное нарушение работы датчика сенсорной сети, что приводит к расходимости традиционных алгоритмов калмановской фильтрации параметров движения источника радиоизлучения. В работе на основе математического аппарата смешанных марковских процессов в дискретном времени синтезированы оптимальный и квазиоптимальный алгоритмы адаптивной фильтрации параметров движения источника радиоизлучения на основе TDOA-измерений сенсорной сети при наличии аномальных измерений. Оптимальный алгоритм описывает эволюцию совместной апостериорной плотноти вероятности вектора параметров движения и переменных переключения, определяющих вид ошибок измерения датчиков сети. В полученном путем линеаризации уравнения измерения квазиоптимальном алгоритме реализован последовательный способ обработки поступающих данных и выполняется гауссовская аппроксимация апостериорной плотности вероятности параметров движения источника радиоизлучения. Для рассмотренного с помощью статистического моделирования примера разработанный квазиоптимальный алгоритм позволяет распознать появление аномальних ошибок измерений с вероятностью, близкой к единице, и устранить их влияние на точность определения параметров движения источника радиоизлучения.
\end{abstract}

Ключевые слова: TDOА-измерения, сенсорная сеть, аномальные измерения, параметры движения источника радиоизлучения, смешанные марковские процессы, алгоритм адаптивнного оценивания.

\section{ВСТУПЛЕНИЕ}

Задача пассивного определения местоположения источников радиоизлучения (ИРИ) находит широкое применение при мониторинге окружающего пространства, ликвидации последствий стихийных бедствий, в интеллектуальных транспортных и охранных системах [1-3]. Местоположение ИРИ может быть определено с помощью беспроводных сенсорных сетей (БСС) [4-7], которые стали важной областью исследований в течение последних лет.
БСС начинают активно применяться в различных областях, таких как: аварийно-спасательные операции, автономное наблюдение и мониторинг промышленных процессов и окружающей среды (мониторинг животного мира), контроль и управление подвижными объектами и др. При выполнении аварийно-спасательных операций БСС обеспечивают возможность определения местоположения членов спасательных команд (например, пожарной команды), а также технических средств (на- 


\section{БИБЛИОГРАФИЧЕСКИЙ СПИСОК}

1. Emery, M.; Denko, M. "IEEE 802.11 WLAN based real-time location tracking in indoor and outdoor environments," Proc. of Canadian Conf. on Electrical and Computer Engineering, CCECE'07, 22-26 Apr. 2007, Vancouver, Canada. IEEE, 2007, p. 1062-1065. DOI: 10.1109/CCECE.2007.271.

2. Xu, E.; Ding, Z.; Dasgupta, S. "Target tracking and mobile sensor navigation in wireless sensor networks," IEEE Trans. Mobile Comput., Vol. 12, No. 1, p. 177-186, Jan. 2013. DOI: $10.1109 /$ TMC.2011.262.

3. Sinclair, A. J.; Lovell, T. A.; Darling, J. "RF localization solution using heterogeneous TDOA," IEEE Aerospace Conf., 7-14 Mar. 2015, Big Sky, USA. IEEE, 2015. DOI: 10.1109/AERO.2015.7119256.

4. Mahfouz, S.; Mourad-Chehade, F.; Honeine, P.; Farah, J.; Snoussi, H. "Target tracking using machine learning and Kalman filter in wireless sensor networks," IEEE Sensors J., Vol. 14, No. 10, p. 3715-3725, Oct 2014. DOI: $\frac{10.1109 / \text { JSEN.2014.2332098. }}{5 . J 10, \Phi . ; ~}$

5. Лю, Ф.; Ли, Х.; Ян, Ч. “Метод оценивания последовательно отсутствующих данных сенсоров с использованием глубокой нейронной сети," Известия вузов. Радиоэлектроника, Т. 61, № 6, с. 336-348, 2018. DOI: $10.20535 / \mathrm{S} 0021347018060043$.

6. Alsheikh, Mohammad Abu; Lin, Shaowei; Niyato, Dusit; Tan, Hwee-Pink. "Machine learning in wireless sensor networks: Algorithms strategies and applications,» IEEE Commun. Surveys \& Tutorials, Vol. 16, No. 4, p. 1996-2018, 2014. DOI: 10.1109/COMST.2014.2320099.

7. Amar, Alon; Leus, Geert. "A reference-free time difference of arrival source localization using a passive sensor array," IEEE Sensor Array and Multichannel Signal Processing Workshop, 4-7 Oct. 2010, Jerusalem, Israel. IEEE, 2010, pp. 157-160. DOI: $\underline{10.1109 / S A M .20}$ 10.5606725

8. Zhang, X.; Huang, J.; Wang, Y.; Zhou, Y. “An efficient estimator for target localization in a multistation redundancy system without matrix inversion," J. Sensors, Vol. 2018, p. 1-12, 2018. DOI: 10.1155/2018/6362939.

9. Rullan-Lara, Jose L.; Sanahuja, Guillaume; Lozano, Rogelio; Salaza, Sergio; Garcia-Hernandez, Ramon; Ruz-Hernandez, Jose A. "Indoor localization of a quadrotor based on WSN: a real-time application," Int. J. Advanced Robotic Systems, Vol. 10, No. 1, 2013. DOI: $\underline{10.5772 / 53748 .}$.

10. El Gemayel, N.; Koslowski, S.; Jondral, F. K.; Tschan, J. "A low cost TDOA localization system: Setup, challenges and results," Proc. of 10th Workshop on Positioning, Navigation and Communication, WPNC, 20-21 Mar. 2013, Dresden Germany. IEEE, 2013, pp. 1-4. DOI: $10.1109 /$ WPNC.2013.6533293. 
11. El Gemayel, Noha; Jakel, Holger; Jondral, Friedrich K. "Error analysis of a low cost TDoA sensor network," Proc. of IEEE/ION Position, Location and Navigation Symp., 5-8 May 2014, Monterey, USA. IEEE, 2014, pp. 1140-1145. DOI: 10.1109/PLANS.2014.6851 484.

12. Wan, Pengwu; Li, Zan; Hao, Benjian. "Time delay estimation of co-frequency signals in TDOA localization based on WSN," Proc. of Int. Conf. on Computer, Information and Telecommunication Systems, CITS, 6-8 Jul. 2016, Kunming, China. IEEE, 2016. DOI: 10.1109/CITS.2016.7546399.

13. Makki, Ahmed; Siddig, Abubakr; Saad, Mohamed; Cavallaro, Joseph R.; Bleakley, Chris J. "Indoor localization using 802.11 time differences of arrival," IEEE Trans. Instrumentation Meas., Vol. 65, No. 3, p. 614-623, Mar 2016. DOI: 10.1109/TIM.2015.2506239.

14. Куприянов, А. И.; Сахаров, А. В. Теоретические основы радиоэлектронной борьбы: Учеб. пособие. М.: Вузовская книга, 2007. 356 с.

15. Сирота, А. А.; Кирсанов, Э. А. “Нейросетевые и статистические алгоритмы оценки координат источника радиоизлучения в многопозиционных радиосистемах при наличии аномальных ошибок измерения первичных параметров," Известия вузов. Радиоэлектроника, Т. 49, № 4, С. 19-27, 2006. URI: http:// radio.kpi.ua/article/view/S0021347006040030.
16. You, He; Jianjuan, Xiu; Xin, Guan. Radar Data Processing with Applications. Wiley, 2016. DOI: 10.100 2/9781118956878.

17. Tovkach, I. O.; Zhuk, S. Ya. "Recurrent algorithm for TDOA localization in sensor networks," J. Aerosp. Technol. Manag., Vol. 9, No. 4, p. 489-494, 2017. DOI: 10.5028/jatm.v9i4.727.

18. Евланов, П. А.; Жук, С. Я. "Комплексирование измерителей с отказами,” Известия вузов. Радиоэлектроника, Т. 33, № 7, с. 40-45, 1990.

19. Тихонов, В. И.; Харисов, В. Н. Статистический анализ и синтез радиотехнических устройств и систем. Учебное пособие для вузов. 3-е изд. М.: Горячая Линия-Телеком, 2014. 607 с.

20. Трифонов, А. П.; Шинаков, Ю. С. Совместное различение сигналов и оценка их параметров на фоне помех. М.: Медиа,. 2012. 264 с.

21. Жук, С. Я. “Синтез цифровых обнаружителей-измерителей смешанных марковских процессов,” Известия вузов. Радиоэлектроника, Т. 32, № 11, с. 31-37, 1989. URI: http://radio.kpi.ua/article/view/S00213 4701989110063.

22. Товкач, И. О.; Жук, С. Я. “Адаптивная фильтрация параметров движения источника радиоизлучения при комплексном использовании данных сенсорной сети, полученных на основе методов TDOA и RSS," Известия вузов. Радиоэлектроника, Т. 60, № 12, c. 685-695, 2017. DOI: 10.20535/S0021347017120020. 